\title{
Nasal high flow therapy in very low birth weight infants with mild respiratory distress syndrome: a single center experience
}

\author{
Antonio Di Mauro ${ }^{1 *}$ D, Manuela Capozza', Sergio Cotugno1', Silvio Tafuri², Francesco Paolo Bianchi²,
} Federico Schettini ${ }^{1}$, Raffaella Panza ${ }^{1}$ and Nicola Laforgia ${ }^{1}$

\begin{abstract}
Background: Pulmonary disorders and respiratory failure represent one of the most common morbidities of preterm newborns admitted to neonatal intensive care units (NICUs). The use of nasal high-flow therapy (nHFT) has been more recently introduced into the NICUs as a non-invasive respiratory (NIV) support.

Methods: We performed a retrospective study to evaluate safety and effectiveness of nHFT as primary support for infants born $<29$ weeks of gestation and/or VLBW presenting with mild Respiratory Distress Syndrome (RDS). The main outcome was the percentage of patients that did not need mechanical ventilation. Secondary outcomes were rate of bronchopulmonary dysplasia (BDP), air leaks, nasal injury, late onset sepsis (LOS), intraventricular hemorrhage (IVH), retinopathy (ROP), necrotizing enterocolitis (NEC), hemodynamically-significant patent ductus arteriosus (PDA) and death.

Results: Sixty-four preterm newborns were enrolled. Overall, 93\% of enrolled patients did not need mechanical ventilation. In a subgroup analysis, $88.5 \%$ of infants $<29$ weeks and $86.7 \%$ of infants ELBW $(<1000 \mathrm{~g} \mathrm{BW})$ did not need mechanical ventilation.

BPD was diagnosed in 26.6\% of preterms enrolled (Mild 20\%, Moderate 4.5\%, Severe 1.5\%). In subgroup analysis, BPD was diagnosed in $53.9 \%$ of newborns with GA $<29$ weeks, in $53.3 \%$ of ELBW newborns and in $11.1 \%$ of small for gestational age (SGA) newborns.

Neither air leaks nor nasal injury were recorded as well as no exitus occurred. LOS, IVH, ROP, NEC and PDA occurred respectively in $16.1 \%, 0 \%, 7.8 \%$, and $1.6 \%$ of newborns.

Conclusions: According to our results, n-HFT seems to be effective as first respiratory support in preterm newborns with mild RDS. Further studies in a larger number of preterm newborns are required to confirm nHFT effectiveness in the acute phase of RDS.
\end{abstract}

Keywords: Nasal high flow therapy, Very low birth weight infants, Neonatal respiratory distress syndrome

\footnotetext{
* Correspondence: antonio.dimauro@uniba.it

${ }^{1}$ Neonatology and Neonatal Intensive Care Unit, Department of Biomedical

Science and Human Oncology, "Aldo Moro" University of Bari, Policlinico

Hospital - Piazza Giulio Cesare n. 11, 70124 Bari, Italy

Full list of author information is available at the end of the article
} 


\section{Background}

Pulmonary disorders and respiratory failure represent one of the most common morbidities of preterm newborns admitted to neonatal intensive care units (NICU) [1].

Over the previous decades, mechanical ventilation (MV) has been a common practice in post-delivery respiratory care and has significantly improved the survival of preterm newborns [2]. However, the association between MV and lung injury has been well documented [3].

Therefore, the interest in non-invasive respiratory support has surged, with the aim of reducing the risk of lung injury and the incidence of BPD $[4,5]$.

Continuous positive airway pressure (CPAP) is the current standard of care for non-invasive respiratory support in very preterm newborns over the immediate postnatal period, when endotracheal intubation is not needed [6].

The use of nasal high-flow therapy (nHFT) has been more recently introduced into the NICU setting with several mechanisms of action proposed [7].

Due to a simple interface and small tapered prongs, nHFT is perceived as easier to use by nursing staff [8], more comfortable for the newborn [9] and advantageous for mother-newborn bonding [10-12], when compared to CPAP.

A recent meta-analysis on effectiveness and safety of nHFT in preterm newborns concluded that it is not different compared to other conventional modes of noninvasive respiratory support [13], but point out that the evidence for efficacy of nHFT in the extreme preterm infants, compared with other modes of NIV, is still poor and needs to be addressed in larger studies.

nHFT can be used in different clinical situations, including primary support in post-delivery respiratory care and as a mode of weaning from either mechanical ventilation or CPAP [14] .

A recent Cochrane Review stated that the vast majority of evidence available concerns the use of nHFT as post-extubation support and that very few trials have included extremely preterm newborns so far [15].

The aim of our study was to evaluate the effectiveness and safety of high-flow nasal cannulae therapy as primary respiratory support for infants born $<29$ weeks of gestation and/or VLBW presenting with mild Respiratory Distress Syndrome (RDS).

\section{Methods}

Since 2014, in the NICU of Department of Biomedical Science and Human Oncology, University of Bari "Aldo Moro", Italy, adopted a protocol in which nHFT (Vapotherm ${ }^{\circledast}$ Precision Flow) substituted nCPAP as the primary mode of non-invasive respiratory support for preterm newborns. NHFT failure was considered as the need of intubation and mechanical ventilation.
This retrospective study shows the results from January 2014 to December 2016 and data were gathered from medical records.

The aim of the study was to evaluate the success rate and the safety of nHFT in our cohort of VLBW and ELBW preterm newborns.

The local Ethics Committee (Comitato Etico Azienda Ospedaliera Policlinico di Bari) approved the study protocol.

Newborns were eligible for the study if they met the following inclusion criteria:

1) Inborn

2) Gestational age $<29$ weeks and/or birth weight $\leq 1500 \mathrm{~g}$;

3) nHFT as first respiratory support for mild Respiratory Distress Syndrome (RDS), defined by the presence of one or more of the following within $1 \mathrm{~h}$ of birth:

- Silverman score $\geq 5$;

- $\mathrm{FiO}_{2}>0.3$ to maintain $\mathrm{SaO}_{2}$ in the range 88-93\%;

- Radiological signs (pulmonary hypoinflation, air bronchogram, hypodiafania of lung fields).

Patients were excluded because of the presence of severe comorbidities, namely: major congenital anomalies, major surgical diseases, genetic-metabolic congenital syndromes.

The following baseline data were collected: antenatal maternal steroids administration, sex, birth weight, gestational age, radiological findings, oxygen saturation $\left(\mathrm{SaO}_{2}\right), \mathrm{FiO}_{2}$ requirements, Silverman score, resuscitation in delivery room, caffeine prophylaxis and need for surfactant administration.

Newborns were initially stabilized in delivery room with n-CPAP set at $5 \mathrm{cmH}_{2} \mathrm{O}$ and positive pressure ventilation of $20-25 \mathrm{cmH} 2 \mathrm{O}$. No sustained ventilation was used, accordingly to 2010 AAP guidelines [16].

When intubation at birth was not needed, neonates were transferred to the Neonatal Intensive Unit on n-CPAP $\left(5 \mathrm{cmH}_{2} \mathrm{O}\right)$ during transportation.

At the NICU, all newborns not intubated and ventilated, were started on HFNC as primary respiratory support. HFNC flow rates vary between 4 and $10 \mathrm{~L} / \mathrm{min}$ titrated according to newborn clinical conditions.

We start at a flow rate of $6 \mathrm{~L} / \mathrm{min}$, modulating according to oxygen requirement, $\mathrm{CO} 2$ retention or work of breathing, as reported in mechanistic literature [17]. According to our weaning protocol, we reduce flow rates by $0.5 \mathrm{~L} / \mathrm{min} 12$ hourly, if required $\mathrm{FiO}_{2}$ was lower than $30 \%$. Respiratory rate, work of breathing, desaturations, apneas and bradycardia have been recorded during weaning. The duration of nHFT, i.e. number of days, was also registered. 
Chest radiograms were acquired at bedside with a portable device (PHILIPS PRACTIX 33 PLUS MOBILE RADIOGRAPHY ${ }^{\circ}$ and classified according to the literature [18].

A prophylactic caffeine citrate therapy (Peyona ${ }^{\circ}$ CHIESI Farmaceutici, Italy, loading dose of $20 \mathrm{mg} / \mathrm{kg}$ and maintenance dose of $5 \mathrm{mg} / \mathrm{kg}$ per day) was routinely started early (within $2 \mathrm{~h}$ of age) in our cohort of preterm infants $<34$ weeks GA and given until 34 to 36 weeks corrected gestational age, if free of any apnea episodes for at least one week [19].

Surfactant (Curosurf ${ }^{\circ}$ CHIESI Farmaceutici, Italy), $200 \mathrm{mg} / \mathrm{kg}$, was administered during nHFT, by INSURE technique, with no pre-medication, if required $\mathrm{FiO}_{2}$ was $>35 \%$ to achieve $\mathrm{SaO}_{2}$ 85-93\% [20]. They were immediately extubated thereafter and reassumed to NHFT.

The aim of the study was to determine the effectiveness of nHFT as primary and unique respiratory support, hence neither n-CPAP nor other types of NIV were considered as an alternative support in case of failure.

Therefore, to assess the success rate and the safety of nHFT in our population, the following outcomes were evaluated:

- Percentage of patients that did not need mechanical ventilation within $72 \mathrm{~h}$ after the start of nHFT, due to one or more of the following:

- arterial or arterialized capillary blood gas analysis showing $\mathrm{pH}<7.2$ and/or $\mathrm{pCO}_{2}>70 \mathrm{mmHg}$;

- $\mathrm{FiO}_{2}>40 \%$ after surfactant administration to maintain $\mathrm{SaO}_{2}$ 85-93\%;

- more than 4 episodes of apneas with spontaneous recovery within $1 \mathrm{~h}$, or more than 2 episodes requiring IPPV within $1 \mathrm{~h}$.

- Rate of Bronchopulmonary dysplasia (BPD), according to Bancalari et Jobe classification [21]

- Rate of adverse events

- Air leaks (pneumothorax/pneumomediastinum)

- Nasal trauma (ulcerations, granulations and vestibular stenosis, and necrosis of the columella)

- Rate of neonates presenting the following diagnoses

$\circ$ Late onset sepsis (LOS)

○ Intraventricular hemorrhage (IVH) or periventricular leukomalacia (PVL)

- Retinopathy of prematurity (ROP)

- Necrotizing enterocolitis (NEC)

- Hemodynamically-significant patent ductus arteriosus (PDA) requiring a pharmacologic treatment

- Deaths

Data of total parenteral nutrition duration, time to reach full enteral feeding (defined as a daily intake of $\geq 140 \mathrm{~mL} / \mathrm{kg} /$ day), time to full suckling feeds and total length of admissions, were also collected to evaluate indirect advantages.

Data were gathered from medical records of patients admitted to the Neonatal Intensive Care Unit (NICU) of the University of Bari from January 2014 to December 2016.

The database was uploaded as an Ms. Excel spreadsheet and data were analyzed by Stata MP11 software.

Data were presented using standard descriptive statistics: categorical variables were reported as percentages, whereas quantitative variables were described as means \pm standard deviations. Chi-squared test was used to perform comparisons between percentages. Test $t$-student was used to perform comparisons between means in independent variables normally distributed, whereas Wilcoxon rank sum test was used to perform comparisons between means in independent variables non-normally distributed and not normalizable.

For quantitative variables the analysis of normality was carried out; variables non-normally distributed have been normalized using logarithms.

Statistical analysis determined significant factors (maternal and neonatal characteristics) related to outcomes (failure of high-flow nasal cannula therapy, duration and settings of high-flow nasal cannula therapy, hospitalization and time to reach full enteral feeding).

For qualitative variables, in logistic regression we calculated the adjusted Odds Ratio (aOR) value with a 95\% confidence interval using the $\mathrm{z}$-score test.

For quantitative variables, in linear regression we calculated the correlation coefficient with a 95\% confidence interval using the student's t-test. In all analyses, a $p$-value of $<0.05$ was considered to be statistically significant.

\section{Results}

\section{Population}

Between January 2014 and December 2016, 838 preterm newborns were admitted to the Neonatal Intensive Care Unit of the University of Bari. Of these, according to inclusion/exclusion criteria, 64 preterms were eventually included (Fig. 1).

Demographics at baseline are described in Table 1.

Twenty-nine preterm neonates met GA and birth weight inclusion criteria, but were excluded because intubated in the delivery room. Those neonates featured a lower mean GA $(26.1 \pm 2.5)$, a lower mean BW $(872.2 \pm 348.9)$ and a worse mean Apgar score at 5 min $(7.4 \pm 1.4)$ than the enrolled neonates ( $p$ value $<0.05$, data not shown).

Gestational age of neonates started on HFNC ranged between $26^{+2}$ and $35^{+3}$ weeks with a median of 29 weeks $(\mathrm{DS}= \pm 2.1)$. Birth weight ranged between 650 and $1495 \mathrm{~g}$ with a mean value of $1166 \pm 241 \mathrm{~g}$. Males and females were equally represented. 

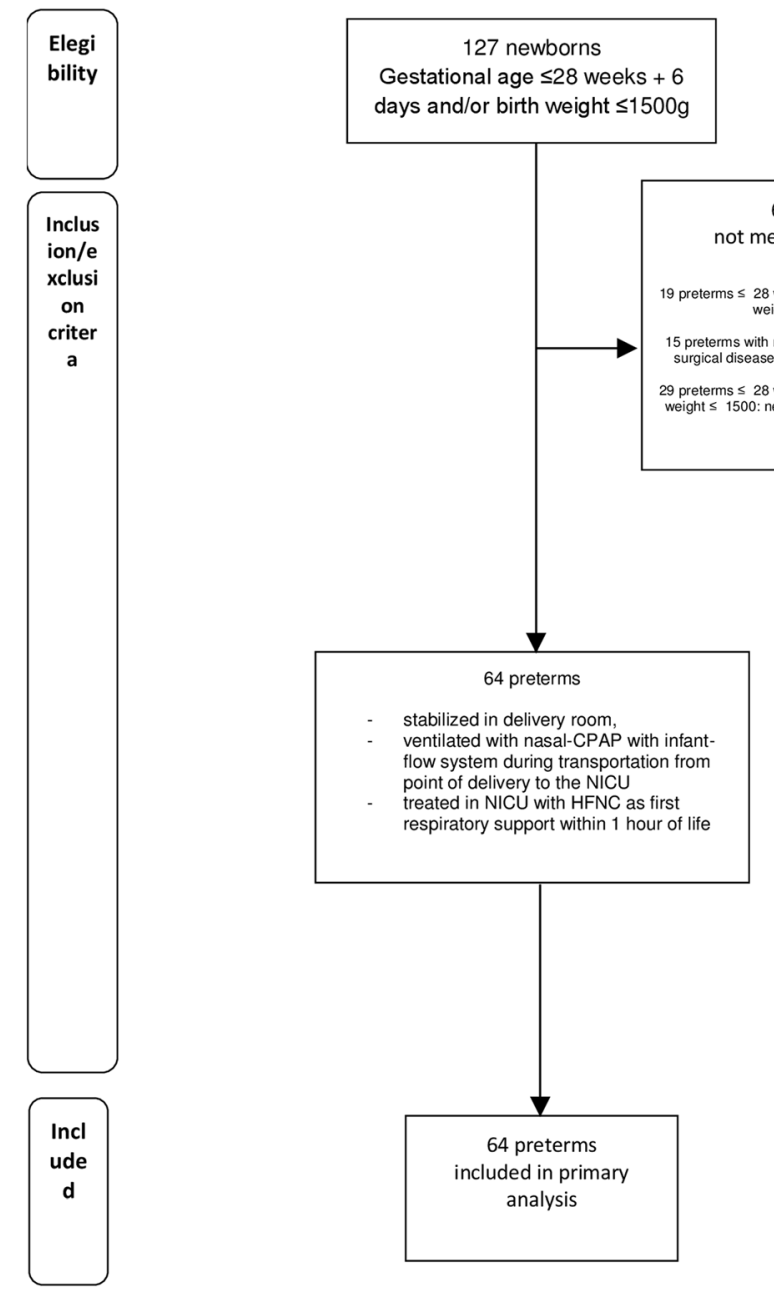

Fig. 1 Flow diagram for the patient recruitment in this study

The mean Silverman Anderson Score at the start of nHFT was $5.7 \pm 1.0$ with a mean $\mathrm{FiO}_{2}$ requirement of $26.4 \pm 4.8 \%$.

According to our protocol 25 (39.1\%) newborns required surfactant administration during treatment. These subgroup median gestational age was 28.5 with a mean birth weight of $1110 \pm 232 \mathrm{~g}$.

During NHFT, the mean value of the maximum flow rates reached $7.3 \pm 0.9 \mathrm{l} / \mathrm{min}$, whereas the mean value of the maximum $\mathrm{FiO}_{2}$ was $28.1 \pm 6.5 \%$. Overall, removing patients who needed mechanical ventilation due to NHFT failure, the median duration of treatment was $72.5 \mathrm{~h}$.

\section{Primary outcome}

The overall success rate, i.e. no mechanical ventilation within $72 \mathrm{~h}$ after nHFT, was 93.7\% (IC 95\%: $85 \%-100 \%$ ). nHFT failed in 4 newborns (median $\mathrm{GA}=27.5 \mathrm{wks}, \mathrm{BW}=965 \pm 169 \mathrm{~g}$ ) at a mean postnatal age of $13.5 \pm 9.9 \mathrm{~h}$ : three of these required surfactant administration during nHFT.
In subgroup analysis, for newborns with $\mathrm{GA}<29$ weeks, the success rate was $88.5 \%\left(X^{2}=2.1 ; p=0.295\right.$; IC 95\%: $70 \%-98 \%)$ and in ELBW newborns, the success rate was $86.7 \%\left(X^{2}=1.7 ; p=0.232\right.$; IC 95\%: $\left.60 \%-98 \%\right)$.

\section{Secondary outcomes}

BPD was diagnosed in 17 newborns (26.6\%; IC 95\%: $16 \%-39 \%)$, three of whom (17.6\%; IC 95\%: $4 \%-43 \%)$ also needed mechanical ventilation.

In subgroup analysis, BPD was diagnosed in $53.9 \%$ (IC 95\%: $33 \%-73 \%$ ) of newborns with $\mathrm{GA}<29$ weeks, in 53.3\% (IC 95\%: 27\% - 79\%) of ELBW newborns and in 11.1\% ( $X^{2}=3.1 ; p=0.117$; IC 95\%: 0.01-0.36) of SGA. BPD occurred more frequently in newborns with a $\mathrm{GA}<29$ weeks compared to those of $\mathrm{GA} \geq 29$ weeks $\left(X^{2}=16.7 ; p=0.000\right)$, and in newborns with a birth weight $<1000 \mathrm{~g}$ compared to those weighing $\geq 1000 \mathrm{~g}\left(X^{2}=7.2 ; p=0.007\right)$. Neither air leaks nor nasal injury were recorded and no exitus occurred.

Regarding morbidities LOS overall incidence was $16.1 \%$. It occurred respectively in $28 \%$ of newborns with 
Table 1 Demographic characteristics of newborns enrolled

\begin{tabular}{|c|c|}
\hline Demographics characteristics & Results \\
\hline \multicolumn{2}{|l|}{ Mothers } \\
\hline Age, m (DS) & $33.7( \pm 5,5)$ \\
\hline Primigravida, n (\%) & $36(56 \%)$ \\
\hline $\begin{array}{l}\text { Antenatal glucocorticoids (either complete } \\
\text { or incomplete), } \mathrm{n}(\%)^{\mathrm{a}}\end{array}$ & $54(84 \%)$ \\
\hline Cesarean Section, n (\%) & $56(87 \%)$ \\
\hline Multiple birth, n (\%) & $24(37 \%)$ \\
\hline High risk pregnancy, n (\%) ${ }^{b}$ & $32(50 \%)$ \\
\hline \multicolumn{2}{|l|}{ Newborns } \\
\hline Gestational age (GA), weeks, m (DS) & $29.4( \pm 2,1)$ \\
\hline \multicolumn{2}{|l|}{ Distribution per week of gestational age (GA } \\
\hline 26 weeks GA, n (\%) & $3(4.5 \%)$ \\
\hline 27 weeks GA, n (\%) & $10(16 \%)$ \\
\hline 28 weeks GA, n (\%) & $13(20 \%)$ \\
\hline 29 weeks GA, n (\%) & $7(11 \%)$ \\
\hline 30 weeks GA, n (\%) & $9(14 \%)$ \\
\hline 31 weeks GA, n (\%) & $8(12.5 \%)$ \\
\hline 32 weeks GA, n (\%) & $10(16 \%)$ \\
\hline 33 weeks GA, n (\%) & $3(4.5 \%)$ \\
\hline 34 weeks GA, n (\%) & $0(0 \%)$ \\
\hline 35 weeks GA, n (\%) & $1(1.5 \%)$ \\
\hline Birth weight, g, m (DS) & $1166( \pm 241)$ \\
\hline VLBW, n (\%) & $49(76 \%)$ \\
\hline ELBW, n (\%) & $15(23 \%)$ \\
\hline SGA, n (\%) & $18(28 \%)$ \\
\hline Males, n (\%) & $32(50 \%)$ \\
\hline Apgar score at 5 min, m (DS) & $8.7( \pm 0,3)$ \\
\hline Neonatal resuscitation, $\mathrm{n}(\%)^{c}$ & $55(85 \%)$ \\
\hline Silverman score at enrollment, m (DS) & $5.7( \pm 1,0)$ \\
\hline $\mathrm{FiO}_{2}$ at enrollment, $\mathrm{m}$ (DS) & $26.3( \pm 4,8)$ \\
\hline $\mathrm{PCO}_{2}$ at enrollment, $\mathrm{m}(\mathrm{DS})$ & $46.9( \pm 7,6)$ \\
\hline pH at enrollment, m (DS) & $7.2( \pm 0,06)$ \\
\hline
\end{tabular}

${ }^{a}$ Antenatal glucocorticoids are medication given to pregnant women expecting a preterm birth that reduce newborn mortality and RDS ${ }^{\mathrm{b}} \mathrm{High}$ risk pregnancy namely clinically diagnosed chorioamnionitis, prolonged premature rupture of membranes greater than $18 \mathrm{~h}$, preeclampsia, and placental abruption

'Oxygen Therapy and Positive pressure ventilation with face mask

GA $<29$ weeks $\left(X^{2}=4.4 ; p=0.07\right)$, in $40 \%$ of ELBW $\left(X^{2}=8.3 ; p=0.009\right)$ and in $11.1 \%$ of SGA $\left(X^{2}=0.5\right.$; $p=0.709)$. ROP, hemodynamically-significant PDA and NEC each occurred in 7.8\% (IC 95\%: 3\% - 17\%), 9.4\% (IC 95\%: 4\% - 19\%), 1.6\% (IC 95\%: 0\% - 8\%) of newborns. No cases of IVH or PVL were reported.

Mean duration of total parenteral nutrition was $13.0 \pm 9.3$ days. Mean time to reach full enteral feeding was $14.6 \pm 9.4$ days and the mean time to full suckling feeds was $32.1 \pm 15.0$ days. Duration of hospitalization was $52 \pm 20$ days. Data on nHFT related outcomes are presented in Table 2.

We also evaluated the relationship between nHFT duration and several maternal and neonatal factors (birth weight, gestational age, weight category for gestational age, delivery mode, gender, maternal age, multiple birth, maternal antenatal steroids, high risk pregnancy, early and late onset sepsis).

In simple linear regression, gestational age (coef. $=-0.30$; $t=4.4 ; p=0.000$; IC 95\%: $-0.43--0.16$ ), birth weight (coef. $-0.001 ; t=2.2 ; p=0.034$; IC 95\% $-0.0027-0.0001$ ), antenatal glucocorticoids (coef. $=0.96 ; \mathrm{t}=2.2 ; p=0.029$; IC 95\%: 0.103-1.820) were positively related to nHFT duration.

In multiple linear regression, only gestational age (coef. $=-0.26 ; t=3.3 ; p=0.002$; IC 95\%: $-0.42--0.10$ ) was positively related to nHFT duration.

Short and long time NHFT failure as well as nHFT duration are not related to $\mathrm{FiO}_{2}$ requirements at baseline $(p>0.05)$.

Lower gestational age neonates are likely to have a higher flow rate $\max$ (coef. $=-1.93 ; t=2.7 ; p=0.010$; IC 95\%: $-3.38--0.47$ ) and a higher $\mathrm{FiO}_{2}$ requirement (coef. $=-0.90 ; t=2.1 ; p=0.038$; IC 95\%: $-1.75--0.05$ ).

Adjusted logistic regression showed that long term failure was positively related to lower gestational age $(\mathrm{aOR}=0.43 ; \mathrm{z}=2.2 ; p=0.025$; IC 95\%: 0.20-0.91).

\section{Discussion}

Over the past several years there has been a growing interest in non-invasive ventilation and nHFT as respiratory support in newborns, despite limited data about its safety and effectiveness in the treatment of extremely preterm newborns [22].

Our study on the efficacy and safety of NHFT, as first respiratory support, in preterm newborns with RDS follows other reports [12, 14, 15, 17-19].

Overall, our success and failure rate of nHFT seem comparable with other randomized clinical trials, assessing NHFT as the primary approach to RDS in newborns greater than 29 weeks GA, compared to nCPAP treatment [23, 24].

Furthermore, this is the first study in newborns with GA $<29$ weeks and ELBW $(<1000 \mathrm{~g})$, showing a subgroup success rate of $88.5 \%$ and $86.7 \%$ respectively. In addition, our data showed that lower gestational ages predict NHFT duration and its long-term failure.

Several studies, although conducted for different purposes and enrolling cohorts of either VLBW or very/ extremely preterm neonates, reported intubation rates in newborns treated with nCPAP after birth, varying from $12.2 \%$ to $52.3 \%$ [25-30]. 
Table 2 Primary and secondary outcomes

\begin{tabular}{|c|c|}
\hline Outcomes & Results \\
\hline \multicolumn{2}{|l|}{ Short-term treatment failure (\%) } \\
\hline Total, n (\%) & $4(6.3)$ \\
\hline$<29$ week, n (\%) & $3(11.5)$ \\
\hline ELBW, n (\%) & $2(13.3)$ \\
\hline SGA, n (\%) & $0(0.0)$ \\
\hline \multicolumn{2}{|l|}{ Long-term treatment failure (\%) } \\
\hline Total, n (\%) & $17(26.6)$ \\
\hline - Mild BDP & $13(20 \%)$ \\
\hline - Moderate BDP & $3(4.5 \%)$ \\
\hline - Severe BDP & $1(1.5 \%)$ \\
\hline$<29$ week, n (\%) & $14(53.9)$ \\
\hline ELBW, n (\%) & $8(53.3)$ \\
\hline SGA, n (\%) & $2(11.1)$ \\
\hline \multicolumn{2}{|l|}{ Adverse event, morbidity, mortality (\%) } \\
\hline Barotrauma and nasal ulceration, n (\%) & $0(0.0)$ \\
\hline Late onset sepsis, n (\%) & $10(16.1)$ \\
\hline $\mathrm{IVH}, \mathrm{n}(\%)$ & $0(0.0)$ \\
\hline $\mathrm{ROP}, \mathrm{n}(\%)$ & $5(7.8)$ \\
\hline NEC, n (\%) & $1(1.6)$ \\
\hline PDA, n (\%) & $6(9.4)$ \\
\hline Exitus, n (\%) & $0(0.0)$ \\
\hline Duration of HFNCT, mean (DS), $\mathrm{h}$ & $141.1( \pm 181.1)$ \\
\hline Median, & 72.5 \\
\hline Min-max & $12-720$ \\
\hline Maximum flow rates, mean (DS), Ipm & $7.3( \pm 0.9)$ \\
\hline Median, & 7,5 \\
\hline Min-max & $4,5-10$ \\
\hline Maximum FiO2, mean (DS), \% & $28.1( \pm 6.5)$ \\
\hline Median, & 25,5 \\
\hline Min-max & $21-50$ \\
\hline Parenteral nutrition duration, mean (DS), days & $13.0( \pm 9.3)$ \\
\hline Median, & 10 \\
\hline Min-max & $2-42$ \\
\hline Time to reach full enteral feeding, mean (DS), days & $14.6( \pm 9.4)$ \\
\hline Median, & 11 \\
\hline Min-max & $6-49$ \\
\hline Time to bottle feed, mean (DS), days & $32.1( \pm 15.0)$ \\
\hline Median, & 33 \\
\hline Min-max & $7-85$ \\
\hline Length of hospitalization, mean (DS), days & $52.4( \pm 20.0)$ \\
\hline Median, & 49 \\
\hline Min-max & $22-94$ \\
\hline
\end{tabular}

Our intubation rate $(6.3 \%)$ was much lower so that NHFT seems comparable to nCPAP to avoid the need for mechanical ventilation.

Our mean flow rates $(7.3 \pm 0.9)$ was higher than other reports $[19,20]$, suggesting that NHFT high flow rates could be used even in very preterm newborns without increasing short term failure rate. However, we have had a remarkable rate of late failure (BPD) in newborns with GA $<29$ weeks and ELBW compared to other studies using nCPAP [31].

Although the data from our retrospective study seem to support the use of NHFT as primary treatment for preterm newborns with mild respiratory distress, the recent multicentre, randomized HIPSTER trial showed significantly higher rates of treatment failure in newborns primarily treated with NHFT than nCPAP, so that nCPAP should be recommended as first-line treatment, even though intubation rates did not differ between NHFT and nCPAP [32].

In preterm newborns treated with nCPAP, the injuries of nasal mucosa or external nares have been reported [33]. In our cohort, no events of nasal trauma occurred, even in the smallest and extreme preterm newborns.

These data are consistent with those published in a recent meta-analysis, which found a lower incidence of nasal trauma in preterm newborns treated with NHFT compared with other forms of NIV [6].

Furthermore, time to reach both full enteral (14.6 \pm 9.4 days) and full suckling feeds (32.1 \pm 15.0 days) were lower in our cohort compared to those reported in VLBW newborns less than 30 weeks gestational age [34].

These two outcomes are strictly related to the lower duration of parenteral nutrition $(13.0 \pm 9.3)$, the lower rate of late onset sepsis (16.1\%), and the shorter hospital stay $(52.4 \pm 20$ days), compared to others [34, 35]. We can speculate that these outcomes are all indirect benefits of NHFT.

We acknowledge several limitations of this study: the retrospective design and the lack of a control group preclude the possibility to make strong assumptions from the presented data on a relatively small sample size.

Our outcome data could be influenced by a large number of SGA newborns (\% of the whole sample), but the calculated short term failure rate, with exclusion of SGA newborns, is $8,7 \%$ (compared to $6.3 \%$ ) while the long term failure rate is $32.6 \%$, compared to $26.6 \%$ of the entire group $(<29$ wks and/or $<1500 \mathrm{~g}$ ).

\section{Conclusions}

This retrospective study shows that use of High Flow Nasal Cannula Therapy might be feasible and safe as first respiratory support for mild Respiratory Distress 
Syndrome (RDS) in preterm newborns with a mean gestational age of 29 weeks.

Further well-designed, prospective and randomized trials are needed before to confirm and recommend the use of HFNC in the treatment of respiratory distress of preterm infants.

\section{Abbreviations \\ BDP: Bronchopulmonary dysplasia; BW: Birth Weight; ELBW: Extremely low birth weight infants; GA: Gestational age; IVH: Intraventricular hemorrhage; MV: Mechanical ventilation; nCPAP: Nasal continuous positive airway pressure; NEC: Necrotizing enterocolitis; nHFT: Nasal high flow therapy; NICU: Neonatal intensive care units; NIV: Non invasive ventilation; PDA: Patent ductus arteriosus; PVL: Periventricular leukomalacia; RDS: Respiratory distress syndrome; ROP: Retinopathy of prematurity; SGA: Small for gestational age; VLBW: Very low birth weight infants}

\section{Acknowledgments}

We thank Prof. Fabio Mosca and Dr. Anna Lavizzari for their thorough review and highly appreciate the comments and suggestions, which significantly contributed to improving the quality of the manuscript.

\section{Funding}

No funding was needed for this study.

\section{Availability of data and materials}

The datasets generated and analysed during the current study are available from the corresponding author on reasonable request.

\section{Authors' contributions}

NL conceptualized and designed the study; led data acquisition, analysis, and interpretation; and reviewed the initial manuscript. He had full access to all of the data in the study and takes responsibility for the integrity of the data and the accuracy of the data analysis. ADM conceptualized and designed the study, made substantial contributions to data interpretation, drafted the initial manuscript and revised the manuscript; FS and RP made substantial contributions to conception and design of the study, drafted the initial manuscript and revised the manuscript. SC and MC made substantial contributions to the collection and analysis of data; ST and FPB made substantial contributions to the analysis and interpretation of data. All authors approved the final manuscript as submitted.

Ethics approval and consent to participate

The study protocol was approved by the local Ethics Committee. Our NICU consent, signed by parents at hospital admission, allows to use any personal data in anonymous form for scientific reason or publication.

\section{Consent for publication}

Not applicable.

\section{Competing interests}

The authors declare that they have no competing interests.

\section{Publisher's Note}

Springer Nature remains neutral with regard to jurisdictional claims in published maps and institutional affiliations.

\section{Author details}

'Neonatology and Neonatal Intensive Care Unit, Department of Biomedical Science and Human Oncology, "Aldo Moro" University of Bari, Policlinico Hospital - Piazza Giulio Cesare n. 11, 70124 Bari, Italy. '2Section of Hygiene, Department of Biomedical Science and Human Oncology, "Aldo Moro" University of Bari, Bari, Italy.
Received: 1 September 2017 Accepted: 11 December 2017

Published online: 28 December 2017

\section{References}

1. Stoll BJ, Hansen NI, Bell EF, Shankaran S, Laptook AR, Walsh MC, et al. Neonatal outcomes of extremely preterm infants from the NICHD neonatal research network. Pediatrics. 2010;126:443-56.

2. Costeloe KL, Hennessy EM, Haider S, Stacey F, Marlow N, Draper ES. Short term outcomes after extreme preterm birth in England: comparison of two birth cohorts in 1995 and 2006 (the EPICure studies). BMJ. 2012;345:e7976.

3. Jobe A. Mechanisms of lung injury and Bronchopulmonary dysplasia. Am J Perinatol. 2016;33:1076-8.

4. de Winter JP, de Vries MAG, Zimmermann Lال Clinical practice : noninvasive respiratory support in newborns. Eur J Pediatr. 2010;169:777-82.

5. Shaffer TH, Alapati D, Greenspan JS, Wolfson MR. Neonatal non-invasive respiratory support: physiological implications. Pediatr Pulmonol. 2012;47:837-47.

6. Cummings JJ, Polin RA, the COMMITTEE ON FETUS AND NEWBORN. Noninvasive respiratory support. Pediatrics. 2016;137:e20153758.

7. Manley BJ, Owen LS. High-flow nasal cannula: mechanisms, evidence and recommendations. Semin Fetal Neonatal Med. 2016;21:139-45.

8. Roberts CT, Manley BJ, Dawson JA, Davis PG. Nursing perceptions of high-flow nasal cannulae treatment for very preterm infants. J Paediatr Child Health. 2014:50:806-10.

9. Osman M, Elsharkawy A, Abdel-Hady H. Assessment of pain during application of nasal-continuous positive airway pressure and heated, humidified high-flow nasal cannulae in preterm infants. J Perinatol Off J Calif Perinat Assoc. 2015;35:263-7.

10. Hough $J$, Shearman AD, Jardine LA, Davies MW. Humidified high flow nasal cannulae: current practice in Australasian nurseries, a survey. J Paediatr Child Health. 2012:48:106-13.

11. Manley BJ, Owen L, Doyle LW, Davis PG. High-flow nasal cannulae and nasal continuous positive airway pressure use in non-tertiary specia care nurseries in Australia and New Zealand. J Paediatr Child Health 2012:48:16-21

12. Klingenberg C, Pettersen M, Hansen EA, Gustavsen LJ, Dahl IA, Leknessund A, et al. Patient comfort during treatment with heated humidified high flow nasal cannulae versus nasal continuous positive airway pressure: a randomised cross-over trial. Arch Dis Child Fetal Neonatal Ed. 2014;99:F134-7.

13. Kotecha SJ, Adappa R, Gupta N, Watkins WJ, Kotecha S, Chakraborty M. Safety and efficacy of high-flow nasal Cannula therapy in preterm infants: a meta-analysis. Pediatrics. 2015;136:542-53.

14. Dani C, Pratesi S, Migliori C, Bertini G. High flow nasal cannula therapy as respiratory support in the preterm infant. Pediatr Pulmonol. 2009;44:629-34.

15. Wilkinson D, Andersen C, O'Donnell CP, De Paoli AG, Manley BJ. High flow nasal cannula for respiratory support in preterm infants. In: The Cochrane Collaboration, editor. Cochrane Database Syst. Rev. [Internet]. Chichester, UK John Wiley \& Sons, Ltd; 2016 [cited 2017 Apr 3]. Available from: http://doi.wiley.com/10.1002/14651858.CD006405.pub3

16. Perlman JM, Wyllie J, Kattwinkel J, Atkins DL, Chameides L, Goldsmith JP, et al. Neonatal resuscitation: 2010 international consensus on cardiopulmonary resuscitation and emergency cardiovascular care science with treatment recommendations. Pediatrics. 2010;126:e1319-44.

17. Miller TL. High Flow Nasal Cannula Therapy in Neonatology. Vapotherm Clinical Information; 2012.

18. Arthur R. The neonatal chest X-ray. Paediatr Respir Rev. 2001:2:311-23.

19. Abdel-Hady H. Caffeine therapy in preterm infants. World J Clin Pediatr. 2015:4:81

20. Rojas MA, Lozano JM, Rojas MX, Laughon M, Bose CL, Rondon MA, et al Very early surfactant without mandatory ventilation in premature infants treated with early continuous positive airway pressure: a randomized, Controlled Trial. Pediatrics. 2009;123:137-42

21. Jobe AH, Bancalari E. Bronchopulmonary dysplasia. Am J Respir Crit Care Med. 2001;163:1723-9.

22. Manley BJ, Dold SK, Davis PG, Roehr CC. High-flow nasal Cannulae for respiratory support of preterm infants: a review of the evidence. Neonatology. 2012;102:300-8.

23. Yoder BA, Stoddard RA, Li M, King J, Dirnberger DR, Abbasi S. Heated, humidified high-flow nasal Cannula versus nasal CPAP for respiratory support in neonates. Pediatrics. 2013;131:e1482-90. 
24. Lavizzari A, Colnaghi M, Ciuffini F, Veneroni C, Musumeci S, Cortinovis I, et al. Heated, Humidified High-Flow Nasal Cannula vs Nasal Continuous Positive Airway Pressure for Respiratory Distress Syndrome of Prematurity: A Randomized Clinical Noninferiority Trial. JAMA Pediatr. [Internet]. 2016

[cited 2017 Apr 3]; Available from: http://archpedi.jamanetwork.com/ article.aspx?doi=10.1001/jamapediatrics.2016.1243.

25. Dunn MS, Kaempf J, de Klerk A, de Klerk R, Reilly M, Howard D, et al. Randomized trial comparing 3 approaches to the initial respiratory Management of Preterm Neonates. Pediatrics. 2011;128:e1069-76.

26. Gonçalves-Ferri WA, Martinez FE, Caldas JPS, Marba STM, Fekete S, Rugolo L, et al. Application of continuous positive airway pressure in the delivery room: a multicenter randomized clinical trial. Braz J Med Biol Res. 2014;47:259-64.

27. Han VK, Beverley DW, Clarson C, Sumabat WO, Shaheed WA, Brabyn DG, et al. Randomized controlled trial of very early continuous distending pressure in the management of preterm infants. Early Hum Dev. 1987;15:21-32.

28. Morley CJ, Davis PG, Doyle LW, Brion LP, Hascoet J-M, Carlin JB. Nasal CPAP or intubation at birth for very preterm infants. N Engl J Med. 2008;358:700-8.

29. Tapia JL, Urzua S, Bancalari A, Meritano J, Torres G, Fabres J, et al. Randomized trial of early bubble continuous positive airway pressure for very low birth weight infants. J Pediatr. 2012;161:75-80. e1.

30. Sandri F, Ancora G, Lanzoni A, Tagliabue P, Colnaghi M, Ventura ML, et al. Prophylactic nasal continuous positive airways pressure in newborns of 28-31 weeks gestation: multicentre randomised controlled clinical trial. Arch Dis Child Fetal Neonatal Ed. 2004:89:F394-8.

31. Sandri F, Plavka R, Ancora G, Simeoni U, Stranak Z, Martinelli S, et al. Prophylactic or early selective surfactant combined with nCPAP in very preterm infants. Pediatrics. 2010;125:e1402-9.

32. Roberts $C T$, Owen $L S$, Manley BJ, Frøisland DH, Donath SM, Dalziel KM, et al. Nasal high-flow therapy for primary respiratory support in preterm infants. N Engl J Med. 2016;375:1142-51.

33. Newnam KM, McGrath JM, Estes T, Jallo N, Salyer J, Bass WT. An integrative review of skin breakdown in the preterm infant associated with nasal continuous positive airway pressure. J Obstet Gynecol Neonatal Nurs JOGNN. 2013:42:508-16.

34. Glackin SJ, O'Sullivan A, George S, Semberova J, Miletin J. High flow nasal cannula versus NCPAP, duration to full oral feeds in preterm infants: a randomised controlled trial. Arch. Dis. Child. - Fetal Neonatal Ed. 2016; fetalneonatal-2016-311388.

35. Cohen-Wolkowiez M, Moran C, Benjamin DK, Cotten CM, Clark RH, Benjamin DK, et al. Early and late onset sepsis in late preterm infants. Pediatr Infect Dis J. 2009;28:1052-6.

\section{Submit your next manuscript to BioMed Central and we will help you at every step:}

- We accept pre-submission inquiries

- Our selector tool helps you to find the most relevant journal

- We provide round the clock customer support

- Convenient online submission

- Thorough peer review

- Inclusion in PubMed and all major indexing services

- Maximum visibility for your research

Submit your manuscript at www.biomedcentral.com/submit

CBiomed Central 